\title{
Transición epitelio-mesenquimal (TEM), proliferación y angiogénesis en cáncer de cérvix localmente avanzado (CCLA)
}

- Leonardo Rojas, Andrés Felipe Cardona, Hernán Carranza, Carlos Vargas, Jorge Otero, Luis Fernando Jaramillo, Pilar Archila, Delma Zea, July Rodríguez, Lucely Cetina

Instituto Javeriano de Oncología, Fundación para la Investigación Clínica y Molecular Aplicada del Cáncer (Ficmac), Bogotá, D.C.

Contacto: a_cardonaz@yahoo.com

Introducción y objetivos. El cáncer de cérvix constituye la segunda causa de muerte por cáncer en los países en vía de desarrollo.

Materiales y métodos. Evaluar la asociación de TEM con la expresión de genes relacionados con proliferación y crecimiento tumoral, y su papel pronóstico en pacientes con CCLA tratadas con quimiorradiación.

Resultados. Se incluyeron 61 mujeres con una edad media de 52 años (DE \pm 10 ); todas tenían CCLA (IIA 2,3\% / IIB 47,5\% / IIIA 4,9\% / IIIB 37,7\% / IVA 3,3\% / no definido 3,3\%) con un volumen tumoral de $6,4 \mathrm{~cm}(\mathrm{DE} \pm 1,8 \mathrm{~cm})$ e infección por $\mathrm{VPH}$ en el $46 \%$. La mediana para la SLP y SG fue de 6,6 (IC95\% 4,0-9, 1) y 30,0 meses (IC95\% 1148), respectivamente. Ninguna de las variables tuvo efecto sobre la
SLP, mientras que los niveles del VEGF $(p=0,026), \operatorname{EGFR}(p=0,030)$ y el volumen tumoral $<6 \mathrm{~cm}(p=0,02)$ influyeron sobre este desenlace. La expresión de Twist y la pérdida de E-cadherina se relacionaron con la expresión de VEGF y EGFR, y también influyeron sobre la SG. Los pacientes que tuvieron $>$ Twist mostraron una menor $S G(p=0,0001)$, al igual que aquellos con pérdida de E-cadherina $(p=0,02)$. Cuando se asoció la positividad para la expresión de EGFR o VEGF con la presencia de marcadores de TEM, la SG fue aún menor $(p=0,02)$.

Conclusiones. La TEM se relaciona con la expresión de genes de proliferación y angiogénesis, y tiene una influencia sobre el pronóstico del CCLA.

\section{Tratamiento del cáncer colorrectal metastásico. Experiencia del Centro Javeriano de Oncología del Hospital Universitario San Ignacio, años 2008 a 2011}

\section{Annie Katherine Natera Melo, Aylen Vanessa Ospina Serrano, Ricardo Bruges Maya, Álvaro Ruiz Morales}

Hospital Universitario San Ignacio, Bogotá, D.C.

Contacto: annienatera@gmail.com

Introducción y objetivos. Describir las características demográficas e histopatológicas de pacientes con cáncer colorrectal metastásico que recibieron quimioterapia en CJO entre 2008 y 2011, tratamientos recibidos, posibilidad de metastasectomía, toxicidad y tasas de sobrevida global y libre de progresión.

Materiales y métodos. Estudio descriptivo y retrospectivo, se obtuvo información sobre características demográficas, histopatológicas y terapéuticas de 126 pacientes. Análisis realizado mediante Stata 13, comparación de variables con test de chi cuadrado, exacto de Fisher y curvas de Kaplan-Meier.

Resultados. El esquema de quimioterapia más utilizado como primera línea incluía oxaliplatino, en líneas subsecuentes fue Folfiri. La sobrevida libre de progresión fue similar en todas las líneas de tratamiento. La mayoría de los pacientes recibió anticuerpo monoclonal, siendo bevacizumab el más utilizado y mostrando mejores tasas de respuesta. El 11\% de los pacientes fue llevado a metastasectomía, la mitad recibió Folfox más bevacizumab previo al procedimiento, evidenciándose mayor sobrevida global al compararla con los pacientes que no recibieron este tratamiento (74 vs. 39 meses, $p=0,001$ ). La toxicidad significativa reportada fue del $21 \%$ con Folfox (neuropatía) y del $11 \%$ con Folfiri (gastrointestinal). Con bevacizumab se registró el $5 \%$ de eventos trombóticos y el 1,8\% de sangrado gastrointestinal.

Conclusiones. Las características demográficas y respuestas tumorales al tratamiento fueron similares a las descritas a nivel mundial. Ningún esquema de quimioterapia mostró superioridad en tasas de respuesta y la toxicidad asociada fue predecible. Las terapias biológicas mostraron una mayor tasa de respuesta al compararlas con quimioterapia convencional y el perfil de toxicidad también fue predecible. El grupo de metastasectomía registró una mayor sobrevida global. 